\title{
DOMINATED ESTIMATES IN HILBERT SPACE
}

\author{
M. A. AKCOGLU ${ }^{1}$ AND H. D. B. MILLER
}

\begin{abstract}
Let $U$ be a unitary operator on a Hilbert space $H$, and let $A_{n}(U)$, $n=1,2, \ldots$, be the Cesaro means of $U$. It is shown that $\sum_{n=1}^{\infty} P_{n} A_{n}(U)$ is bounded for every sequence of mutually orthogonal projections $P_{n}, n=1$, $2, \ldots$, if and only if 1 is not a limit point of the spectrum of $U$. The proof is obtained by adapting ideas of Menchoff and Burkholder to show that for any orthonormal sequence $f_{n}, n=0, \pm 1, \pm 2, \ldots$, in $H$, there is an orthonormal sequence $g_{n}, n=1,2, \ldots$, such that
\end{abstract}

$$
\sum_{k=1}^{n}\left|\left(f_{1}+f_{2}+\cdots+f_{k}, g_{k}\right)\right|^{2} \geqslant \frac{1}{36} n(\log n)^{2}
$$

1. Introduction. Let $T: H \rightarrow H$ be a bounded linear operator on a Hilbert space $H$ and let $A_{n}(T)=n^{-1} \sum_{k=0}^{n-1} T^{k}$, where $n$ ranges through the integers $\{1,2 \ldots\}$. If the operator $\sum_{n=1}^{\infty} P_{n} A_{n}(T)$ is a bounded operator for every choice of the mutually orthogonal projections $\left(P_{n}\right)$, then we will say that $T$ admits a strong dominated estimate. The main purpose of this note is to show that a unitary operator $U: H \rightarrow H$ admits a strong dominated estimate if and only if $z=1$ is an isolated point of its spectrum.

This definition of strong dominated estimates was implicitly given in [1], in connection with the usual dominated estimates in ergodic theory. In [1] it was shown that if unitary operators admit strong dominated estimates, then the same would also be true for contractions, and that this would give the dominated ergodic estimate for contractions on $L_{2}$-spaces. It turned out, however, that a result in Burkholder's theory of semi-Gaussian spaces [2] already implied that not all contractions (consequently, not all unitary operators) admit a strong dominated estimate. This implication had the nature of an existence proof, depending on the fact that there are series of orthogonal functions in $L_{2}$-spaces, whose partial sums are convergent in $L_{2}$-norm but not a.e., as was shown by Menchoff [3]. It seemed very desirable to isolate the parts of the arguments in [3] and [2] that are related to strong dominated estimates and to combine these parts to give a direct, purely Hilbert space theoretic proof that not all unitary operators admit a strong dominated estimate. The present note is a result of this program.

2. The main result. For the 'if' part of the assertion, note that, if $z=1$ is an isolated point of the spectrum of $U$, then there is a number $\theta>0$ such that $\left\{e^{i t}: 0<|t|<\theta\right\}$ has spectral measure zero. Hence, we may write $H=H_{1} \oplus$

Received by the editors June 11, 1974.

AMS (MOS) subject classifications (1970). Primary 46C05, 47A35, 47B15.

'Research supported by NRC Grant A3974. 
$H_{2}$ where $U x=x$ for $x \in H_{1}$ and where $\left\|A_{n}(U) x\right\| \leqslant(B / n)\|x\|$ for $x \in H_{2}$ and $B=1 / \sin (\theta / 2)$.

If $P_{n}, n=1,2, \ldots$, is any mutually orthogonal system of projections, then if $x \in H$ and $x=x_{1}+x_{2}, x_{1} \in H_{1}, x_{2} \in H_{2}$, we have

$$
\begin{aligned}
\left\|\sum_{n=1}^{\infty} P_{n} A_{n}(U) x\right\| & \leqslant\left\|\sum_{n=1}^{\infty} P_{n} x_{1}\right\|+\left\|\sum_{n=1}^{\infty} P_{n} A_{n}(U) x_{2}\right\| \\
& \leqslant\left\|x_{1}\right\|+B\left(\sum_{n=1}^{\infty} n^{-2}\right)^{1 / 2}\left\|x_{2}\right\| .
\end{aligned}
$$

Thus,

$$
\left\|\sum_{n=1}^{\infty} P_{n} A_{n}(U)\right\| \leqslant M
$$

where

$$
M=1+B \cdot\left(\sum_{n=1}^{\infty} n^{-2}\right)^{1 / 2}<\infty .
$$

For the 'only if' part of the result, assume that $z=1$ is not an isolated point of the spectrum of $U$. Then there is an orthonormal sequence $e_{n}, n=1$, $2, \ldots$, in $H$ so that

(i) for each $m, \lim _{n \rightarrow \infty}\left\|A_{n}(U) e_{m}\right\|=0$,

(ii) for each $n, \lim _{m \rightarrow \infty}\left\|A_{n}(U) e_{m}-e_{m}\right\|=0$.

Indeed, let $\left(\theta_{m}\right)$ be a sequence so that $\pi>\theta_{m}>\theta_{m+1}>0$ and $\lim _{m \rightarrow \infty} \theta_{m}$ $=0$. We may assume that the spectral measure of $E_{m}=\left\{e^{i \theta} \mid \theta_{m} \geqslant \theta\right.$ $\left.>\theta_{m+1}\right\}$ is nonzero for each $m$. Choose an orthonormal sequence $\left(e_{m}\right)$ in $H$ so that in the spectral representation of $U$, the vector $e_{m}$ corresponds to a function with support in $E_{m}$. Hence,

$$
\left\|A_{n}(U) e_{m}\right\| \leqslant \sup _{z \in E_{m}}\left|\frac{1}{n} \sum_{k=0}^{n-1} z^{k}\right| \leqslant \frac{1}{n \sin \left(\theta_{m+1} / 2\right)},
$$

and

$$
\left\|A_{n}(U) e_{m}-e_{m}\right\| \leqslant \sup _{z \in E_{m}}\left|\frac{1}{n} \sum_{k=0}^{n-1}\left(z^{k}-1\right)\right| \leqslant \frac{2}{n} \sum_{k=0}^{n-1} \sin \frac{(k+1) \theta_{m}}{2},
$$

from which assertions (i) and (ii) follow.

The desired result now follows from Theorem 2.1 below.

(2.1) THEOREM. Let $e_{n}, n=0, \pm 1, \pm 2, \ldots$, be an orthonormal sequence in $H$, and let $R_{n}, n=1,2, \ldots$, be a sequence of bounded linear operators on $H$ such that

(i) for each $m \geqslant 1, \lim _{n \rightarrow \infty}\left\|R_{n} e_{m}\right\|=0$;

(ii) for each $n, \lim _{m \rightarrow \infty}\left\|R_{n} e_{m}-e_{m}\right\|=0$.

Then there is a system $P_{n}, n=1,2, \ldots$, of mutually orthogonal projections in $H$ such that $\lim _{n \rightarrow \infty}\left\|\sum_{k=1}^{n} P_{k} R_{k}\right\|=\infty$.

Proof. Let $P_{n}, n=1,2, \ldots$ be a sequence of mutually orthogonal projections; $\left\|\sum_{j=1}^{n} P_{j} R_{j}\right\|$ is an increasing function of $n$. 
Let $m(j), j=1,2, \ldots$, and $n(j), j=1,2, \ldots$, be any strictly increasing sequences of positive integers. Let the closed subspace spanned by $e_{m(j)}$, $j=1,2, \ldots$, be $K$ and the projection on $K$ be $E$.

Let $T_{k}: H \rightarrow K$ be given by

$$
T_{k} x=\sum_{i=k+1}^{\infty}\left(x, e_{m(i)}\right) e_{m(i)} ;
$$

let $S_{k}=E-T_{k}$. Then

$$
\begin{gathered}
\left\|\sum_{k=1}^{l} P_{n(k)} R_{n(k)} E\right\| \\
\|\| \sum_{k=1}^{l} P_{n(k)} R_{n(k)} E\|-\| \sum_{k=1}^{l} P_{k} P_{k} \| ; \\
\leqslant\left\|\sum_{k=1}^{l} P_{n(k)}\left(R_{n(k)}+S_{k}\right) E\right\| .
\end{gathered}
$$

Thus, we obtain the theorem from the following lemmas.

(2.2) Lemma. The subsequences $m(j), j=1,2, \ldots$ and $n(j), j=1,2, \ldots$, may be chosen so that for $l \geqslant 1$,

$$
\left\|\sum_{k=1}^{l} P_{n(k)}\left(R_{n(k)}+S_{k}\right) E\right\|<2 .
$$

(2.3) Lemma. If $f_{n}, n=0, \pm 1, \pm 2, \ldots$, is an orthonormal sequence in $H$, there is an orthonormal sequence $g_{n}, n=1,2, \ldots$, such that for $n \geqslant 1$,

$$
\left\|\sum_{k=1}^{n} P_{k} \bar{S}_{k}\right\|>\frac{1}{12 \sqrt{3}} \log n,
$$

where $\bar{S}_{k} x=\sum_{i=1}^{k}\left(x, f_{i}\right) f_{i}$ and $P_{k} x=\left(x, g_{n}\right) g_{n}$.

Proof (2.2). First let $m(j)=n(j)=j, j=1,2 \ldots$ Then $T_{n} e_{m}=0$ if $n \geqslant m$ and $T_{n} e_{m}=e_{m}$ if $m \geqslant n+1$. Thus (i), (ii) become

$$
\lim _{n \rightarrow \infty}\left\|\left(R_{n}-T_{n}\right) e_{m}\right\|=0, \text { and } \lim _{m \rightarrow \infty}\left\|\left(R_{n}-T_{n}\right) e_{m}\right\|=0 \text {. }
$$

By induction one can find $m(j), j=1,2, \ldots$, and $n(j), j=1,2, \ldots$, such that if $j, k \geqslant 1$,

$$
\left\|\left(R_{n(j)}-T_{j}\right) e_{m(k)}\right\| \leqslant 2^{-(j+k)} .
$$

We have then

$$
\left\|\left(R_{n(j)}-T_{j}\right) E\right\| \leqslant 2^{-j},
$$

and since

$$
\begin{aligned}
\sum_{j=1}^{l} P_{n(j)}\left(R_{n(j)}+S_{j}\right) E= & \sum_{j=1}^{l} P_{n(j)}\left(R_{n(j)}-T_{j}\right) E \\
& +\sum_{j=1}^{l} P_{n(j)} E,
\end{aligned}
$$

the lemma follows. 
Proof (2.3). It is enough to show that there is an orthonormal sequence $g_{n}$, $n=1,2, \ldots$, such that for all $n \geqslant 1$,

$$
\sum_{k=1}^{n}\left|\left(f_{1}+\cdots+f_{k}, g_{k}\right)\right|^{2} \geqslant \frac{1}{36} n(\log n)^{2} .
$$

(Indeed, given such a sequence, let $n \geqslant 1$, and let $x=\left(\sum_{i=1}^{n} f_{i}\right) / \sqrt{n}$; then $\|x\|=1$, and

$$
\left\|\sum_{k=1}^{n} P_{k} \bar{S}_{k} x\right\|^{2}=\sum_{k=1}^{n}\left|\left(\frac{f_{1}+\cdots+f_{k}}{\sqrt{n}}, g_{k}\right)\right|^{2},
$$

which gives the lemma.)

To show this, put $a(j)=1 /\left(j+\frac{1}{2}\right), j=0, \pm 1, \pm 2, \ldots$ Then

(1) if $l \geqslant 1, \sum_{j=-\infty}^{\infty} a(j) a(j+l)=0$;

(2) $8 \leqslant \sum_{j=-\infty}^{\infty}(a(j))^{2} \leqslant 12$;

(3) if $k \geqslant 1, \sum_{j=0}^{k-1} a(j) \geqslant \log (k+1)$.

((1) follows by writing $a(j) a(j+l)=l^{-1}(a(j)-a(j+l))$, and (3) follows from the inequality (valid if $j \geqslant 0$ )

$$
\left.a(j) \geqslant \int_{j+1}^{j+2} x^{-1} d x=\log (j+2)-\log (j+1) .\right)
$$

For $k \geqslant 1$, let $g_{k}^{\prime}=\sum_{j=-\infty}^{\infty} a(j-k) f_{k}$, and $g_{k}=g_{k}^{\prime} /\left\|g_{k}^{\prime}\right\|$. From (1), (2), (3) it follows that

(4) $g_{k}, k=1,2, \ldots$, is an orthonormal sequence;

(5) if $k \geqslant 1,\left(f_{1}+\cdots+f_{k}, g_{k}\right) \geqslant 12^{-1 / 2} \log (k+1)$.

Thus, if $n \geqslant 1$,

$$
\sum_{k=1}^{n}\left|\left(f_{1}+\cdots+f_{k}, g_{k}\right)\right|^{2} \geqslant \frac{1}{12} \sum_{k=1}^{n}(\log (k+1))^{2} .
$$

The desired result follows by observing that

$$
\log (k+1) \geqslant(k / n) \log (n+1) \text { for } 1 \leqslant k \leqslant n
$$

(which can be proved by making the substitution $x=k(u-1) / n+1$ in $\left.\int_{1}^{k+1} x^{-1} d x\right)$; and that

$$
\sum_{k=1}^{n} k^{2}=n(n+1)(2 n+1) / 6 \geqslant n^{3} / 3 .^{2}
$$

3. Further remarks. Theorem (2.1) may also be applied to some other cases. For example, if $T: H \rightarrow H$ has the form $T x=\sum_{i=1}^{\infty} \lambda_{i}\left(x_{i}, e_{i}\right) e_{i}$ for some orthonormal sequence $e_{i}, i=1,2, \ldots$, such that $0 \leqslant \lambda_{i} \uparrow 1$, then clearly (i), (ii) of the Theorem are satisfied for $e_{n}, n=1,2, \ldots$, and $R_{n}=T^{n}, n=1$, $2, \ldots$, or $R_{n}=I+T+\cdots+T^{n-1} / n, n=1,2 \ldots$

Finally, note that if $U$ is a unitary operator such that $z=1$ is not an isolated point of the spectrum of $U$, then $U$ may be represented on an $L_{2}$ space as a unitary operator for which the dominated ergodic estimate fails. That is, if ' $W$ ' denotes the operator $U$ represented on $L_{2}$, then there is no number $K>0$ such that for all $f \in L_{2},\left\|A^{*} f\right\|<K\|f\|$ : there $A^{*} g$ is defined for $g \in L_{2}$ by

\footnotetext{
${ }^{2}$ The function $a(j)$ (and the properties thereof) is taken from [4, pp. 89-90].
} 


$$
A^{*} g(x)=\sup _{n>1}\left|A_{n}(W) g(x)\right| .
$$

To prove this, let $P_{n}, n=1,2, \ldots$, be a sequence of mutually orthogonal projections such that $\lim _{n \rightarrow \infty}\left\|\sum_{k=1}^{n} P_{k} A_{k}(U)\right\|=\infty$. We may choose $P_{k}$ so that $P_{k} x=\left(x, g_{k}\right) g_{k}$, where $g_{k} \in H$ and $\left\|g_{k}\right\|=1$ if $P_{k} \neq 0$. Choose an isomorphism of $H$ with an $L_{2}$ such that $g_{k}$ is represented as $c_{k} \chi\left(E_{k}\right)$, where $E_{k}$ is a measurable set, and $c_{k}$ is a real number. Then if $x \in H$ corresponds to $f \in L_{2}$, we have

$$
\begin{aligned}
\left\|\sum_{k=1}^{n} P_{k} A_{k}(U) x\right\|^{2} & =\sum_{k=1}^{n}\left|\int c_{k} \chi\left(E_{k}\right) A_{k}(W) f\right|^{2} \\
& \leqslant \sum_{k=1}^{n} \int\left|\chi\left(E_{k}\right) A_{k}(W) f\right|^{2} \leqslant \sum_{k=1}^{n} \int \chi\left(E_{k}\right) \cdot\left|A^{*} f\right|^{2} .
\end{aligned}
$$

Thus, since the $E_{k}$ are disjoint,

$$
\left\|A^{*} f\right\| \geqslant\left\|\sum_{k=1}^{n} P_{k} A_{k}(U) x\right\| .
$$

For some $x \in H$ with $\|x\|=1$, we have

$$
\left\|\sum_{k=1}^{n} P_{k} A_{k}(U) x\right\|>\frac{1}{2}\left\|\sum_{k=1}^{n} P_{k} A_{k}(U)\right\| \text {. }
$$

It follows there is, for each $n \geqslant 1$, an $f \in L_{2},\|f\|=1$, for which

$$
\left\|A^{*} f\right\|>\frac{1}{2}\left\|\sum_{k=1}^{n} P_{k} A_{k}(U)\right\| .
$$

This gives the stated result.

\section{REFERENCES}

1. M. A. Akcoglu and L. Sucheston, On the dominated ergodic theorem in $L_{2}$ space, Proc. Amer. Math. Soc. 43 (1974), 379-382.

2. D. L. Burkholder, Semi-Gaussian subspaces, Trans. Amer. Math. Soc. 104 (1962), 123-131. MR 25 \#2426.

3. D. Menchoff, Sur les séries de fonctions orthogonales, Fund. Math. 4 (1923), 82-105.

4. G. Alexits, Konvergenzprobleme der orthogonalreihen, Akad. Kiado, Budapest, 1960; English transl., Internat. Series Monographs Pure and Appl. Math., vol. 20, Pergamon Press, New York, 1961. MR 28 \#292; 36 \#1911 (pp. 88-99 contain an exposition of the results of [3]).

Department of Mathematics, University of Toronto, Toronto, Ontario M5S 1A1, Canada 